\section{Sonographisches und klinisches Hüftdysplasie-/Luxationsscreening}

Die sonographische Untersuchung der Säuglingshüfte ist im deutschsprachigen Raum mittlerweile mehr als $15 \mathrm{Jah}-$ re etabliert. Dennoch wird die Methode international immer noch heftig diskutiert. Vor allem im anglo-amerikanischen Sprachraum wird die Effizienz der sonographischen Untersuchung in Zweifel gestellt. Catterall (1994) hält das „unidentifizierbare Rauschen“ des sonographischen Bildes für wenig aussagekräftig und die klinischen Untersuchungsprogramme nach wie vor für überlegen.

Dem stehen tägliche Erfahrungen im deutschsprachigen Raum gegenüber. Es besteht kein Zweifel daran, daß seit Einführung der Ultraschalluntersuchung ein deutlicher Rückgang der späterkannten Hüftgelenksluxationen zu verzeichnen ist (Falliner und Hahne, 1998). Screening-Programme aus Österreich belegen einen Rückgang der Behandlungsrate für Kinder mit konservativen Maßnahmen von ca. $13 \%$ vor Einführung der sonographischen Untersuchung auf ca 6,5\% für 1994 (Grill und Müller, 1997). Gleichzeitig ging auch die Anzahl operativer Eingriffe zurück.

Die Zahlen sind im deutschsprachigen Raum so deutlich, daß Vertreter der Deutschen Gesellschaft für Orthopädie und Traumatologie und der Deutschen Gesellschaft für Kinderheilkunde und Jugendmedizin in Zusammenarbeit mit dem Zentralinstitut für die kassenärztliche Versorgung die Kostenträger davon überzeugen konnten, ein klinisches und sonographisches Screening-Programm auch in der BRD einzuführen. Der Weg bis zur definitiven Etablierung des Programmes war steinig. denn es galt, sich widerstrebende Interessen zu ordnen und einen vernünftigen Konsensus zustande zu bringen. Orthopäden und Pädiater haben für zwei sonographische Untersuchungen, unmittelbar nach der Geburt und in der 4. bis 6. Lebenswoche, plädiert. Da in den Jahren vor 1996 eine große Anzahl von Kindern vielfach ohne Änderung eines therapeutischen Konzeptes sonographiert wurde, waren die Kostenträger hiervon nicht zu überzeugen. Es entstand der Kompromiß einer einmaligen sonographischen Untersuchung als fester Bestandteil des Früherkennungsprogrammes im Kindesalter bei der U3. Klinisch oder anamnestisch auffällige Risikokinder sollten aber bereits spätestens bei der U2 einer Ultraschalluntersuchung unterzogen werden. Dies wurde ebenso wie die empfohlene Therapie in Abhängigkeit von Alter und Ausgangsbefund in Leitlinien festgelegt (Niethard et al. 1997)

Diese Art eines klinischen und sonographischen Hüftdysplasie-Screenings ist neu und noch nicht evaluiert. Dem Programm wurde daher zum Teil mit großer Skepsis begegnet. Wesentliche Kritikpunkte sind, daß auf die Ultraschalluntersuchung unmittelbar nach Geburt nicht verzichtet werden könne, daß eine Kontrolluntersuchung unbedingt notwendig sei und daß der beta-Winkel neben dem alpha-Winkel unbedingt für die Beurteilung herangezogen werden müsse. Ein selten vorgebrachter aber sehr wichtiger Kritikpunkt ist, daß mit dem Screening-Programm die sonographische Untersuchung überwiegend in die Praxen von Kinderärzten und Orthopäden verlagert

Z. Orthop. 136 (1998) 485-486

(C) 1998 F. Enke Verlag Stuttgart wurde, wo keine Ausbildung der angehenden Fachärzte mehr stattfinde. Dementsprechend wäre eine Verschlechterung des Kenntnisstandes und der Qualität sonographischer Untersuchung zu befürchten.

Die Vertreter der wissenschaftlichen Fachgesellschaften haben mit der Etablierung des Screening-Programmes festgeschrieben, daß dieses in dieser Form erstmals laufende Programm mindestens über ein Jahr wissenschaftlich beobachtet wird. Erste Ergebnisse dieser Studien liegen nun vor und werden in diesem Heft der Zeitschrift für Orthopädie Ergebnissen und Erfahrungen aus anderen Kliniken gegenübergestellt. Die Ergebnisse des Jahres 1996, d.h. des ersten Jahres des Screening-Programmes, sind verständlicher Weise Ausdruck der „learning curve“, der für Orthopäden neu etablierten Früherkennungsuntersuchung im Kindesalter. Im Jahr 1996 wurden die zum Zeitpunkt der U3 durchgeführten hüftsonographischen Screening-Untersuchungen $\mathrm{zu} 70 \%$ von Pädiatern, zu $26 \%$ von Orthopäden und von übrigen Arztgruppen zu $4 \%$ erbracht (Altenhofen et al. 1998). Auffällig sind unterschiedliche Interpretationen von Pädiatern und Orthopäden hinsichtlich der Diagnose, aber auch der Therapieempfehlungen. Nur bei $73 \%$ aller untersuchten Kinder entsprechen die aufgeführten diagnostischen und therapeutischen Maßnahmen den Empfehlungen der Leitlinie In $5,4 \%$ wird zu wenig und immerhin in $21,6 \%$ zu viel an Diagnose und Therapie vorgesehen. Während die Pädiater bei jedem 5 Untersuchungsfall über die Leitlinie hinausgehende Kontroll- und Therapieempfehlungen abgeben, sprechen sich die Orthopäden bei jedem 3. Fall für Maßnahmen aus, die von der Leitlinie nicht vorgesehen sind. Derartige Unsicherheiten bei der Therapieempfehlung erklären sich offensichtlich aus der Diskrepanz zwischen der sonographischen Klassifikation der Säuglingshüfte nach Graf und der altersabhängigen Entwicklung des alpha-Winkels wie er von Tschauner et al. beschrieben wurde (1994). Handelt es sich beispielsweise um Kinder mit Hüften des Types IIb, d.h. besteht im 3 Lebensmonat eine Reifungsverzögerung und liegt zugleich ein Risikomerkmal vor, so legt Graf eine (vorübergehende) Therapie nahe (Graf, 1993). Diese Empfehlung steht allerdings nicht im Einklang mit der sogenannten Reifungskurve, die entsprechende alpha-Winkel lediglich als kontrollbedürftig ausweist. Auch die Zusammenstellungen von Falliner und Hahne (1998), Rühmann et al. und Mittelmeier et al. zeigen, daß keine einheitlichen Vorstellungen hinsichtlich der Hüftreifung innerhalb der wichtigen ersten 4 bis 6 Lebenswochen bestehen. Es ist daher unerläßlich, daß die von den Fachgesellschaften in Zusammenarbeit mit den Inauguratoren der Methode entwickelten Leitlinien nochmals überprüft werden.

Kann diese Unsicherheit durch eine zweite bereits unmittelbar nach der Geburt durchgeführte sonographische Untersuchung beseitigt werden? Rühmann und Mitarbeiter sind der Meinung, daß ein generelles sonographisches Neugeborenen-Screening zu fordern sei, wenngleich dies durch die von ihnen vorgelegten Zahlen nicht belegt werden kann. Aufgrund ihrer Erfahrungen in Österreich schlagen Grill und Müller wegen der erheblichen biologischen Schwankungsbreite des alpha-Winkels unmittelbar nach Geburt und auch aus ökonomischen Gesichtspunkten das Ultraschall-Screening zwischen der 4. bis 6 . Lebenswoche vor, wie es in der BRD derzeit gehandhabt wird. Die Zahlen aus der BRD zeigen, daß immerhin $26 \%$ der bei der U3 examinierten Kinder schon vorher untersucht wurden (Günther et al. 1998). Dies ist weit mehr als es gemäß der Häufigkeit der Risikofaktoren in dieser 
Gruppe zu erwarten wäre (Rühmann et al. 1998). Leider haben im Jahr 1996 aber nur 33\% des betreffenden Jahrganges an der U3 teilgenommen (Altenhofen et al. 1998). Dies ist eine der Ursachen dafür, daß bei dem kurzen Beobachtungszeitraum eine Abnahme der stationär behandlungsbedürftigen Hüftgelenksdysplasien und -luxationen noch nicht zu beobachten ist (Günther et al. 1998). Allerdings ist auffällig, daß die Hälfte der von der Evaluationsgruppe erhobenen Hüftgelenksluxationen zeitgerecht klinisch und sonographisch untersucht worden war. Diese Beobachtung wird von Falliner und Hahne unterstützt, die in 6 Jahren ebenfalls bei etwa $50 \%$ der von ihnen behandelten Hüftgelenksluxationen keine Fehler beim sonographischen Screening feststellen konnten. Die Autoren vermuten, daß das Screening-Programm zu einem deutlichen Rückgang der dysplastischen Hüftgelenke (sog. angeborene Hüftgelenksluxationen) führt, während die echten an- geborenen Luxationen verbleiben, die therapeutisch aufwendig und zum Teil auch therapierefraktär sind. Dies wurde auch von Catterall vermutet (1994).

Die Gesamtschau der Studien zeigt, daß das klinische und sonographische Hüftdysplasie-Screening wirkt und daß bei frühzeitiger Behandlung nach mittelfristiger Beobachtungszeit kaum Residualdysplasien oder die früher häufig auftretenden Hüftkopfnekrosen zu beobachten sind (Deimel et al. 1998). Von einer Ausrottung der Hüftgelenksdysplasie kann dagegen bisher nicht die Rede sein. Hierfür ist eine Intensivierung von Kontrollmaßnahmen der Struktur- und Prozeßqualität in der Diagnostik und Behandlung von Hüftreifungsstörungen ebenso wie eine weitere Evaluation des Screening-Programmes zu fordern Günther et al. 1998).

F. U. Niethard, Aachen

\section{Literatur}

Altenhofen, L., P. G. Allhoff, F. U. Niethard: Hüftsonographie-Screening im Rahmen der U3 - Erste Erfahrungen. Z. Orthop. 136 (1998) 501-507

Catterall, A.: The early diagnosis of congenital dislocation of the hip. J. Bone Joint Surg. 76-B (1994) 515-516

Mittelmeier, H., D. Deimel, B. Beger, : Aus einem hüftsonographischen Screening-Programm - Mittelfristige Ergebnisse nach Spreizhosentherapie. Z. Orthop. 136 (1998) 513-518

Falliner, A., H. J. Hahne: Die sogenannte angeborene Hüftgelenksluxation - Entwicklung von Häufigkeit, Diagnose und Therapie in den letzten 7 Jahren. Z. Orthop. 136 (1998) 487-491

Graf, D.: Sonographie der Säuglingshüfte und therapeutische Konsequenzen. Ein Kompendium. Enke, Stuttgart 1993

Grill, F., D. Müller: Hüftsonographisches Screening in Österreich. Orthopädie (1997) 25-32

Günther, P. P., S. Stoll, A. Lorani, F. U. Niethard, L. Altenhofen, Ch. Melzer, R. v. Kries: Erste Ergebnisse aus der Evaluationsstudie des sonographischen Hüftscreenings in der BRD. Z. Orthop. 136 (1998) 508-512

Niethard, F. U., S. Stoll, R. v. Kries: Verhindert das Ultraschallscreening tatsächlich das Auftreten späterkannter und damit Repositionsund/oder -operationspflichtiger Hüftdysplasien/-luxationen? Orthopädie (1997) 367-372

Rühmann, O., W. Konermann, D. Lazovic, L. Vitek, P. Bouklas: Sonographisches Neugeborenen-Screening: Der Einfluß anamnestischer Risikofaktoren auf die Hüftdysplasie. Z. Orthop. 136 (1998) 492-500

Tschauner, W., A. Klapsch, C. Baumgartner, R. Graf: „Reifungskurve“ des sonographischen Alpha-Winkels nach Graf unbehandelter Hüftgelenke im ersten Lebensjahr. Z. Orthop. 132 (1994) 502-504 